\title{
Treatment of uveitis associated with systemic autoimmune diseases in adults: a concise overview
}

\author{
Laura Muntean, Simona Rednic \\ "Iuliu Hatieganu" University of Medicine and Pharmacy, Rheumatology Clinic, Cluj-Napoca, Romania
}

\begin{abstract}
Uveitis represents a heterogenous group of inflammatory ocular diseases which may cause vision loss. Association of uveitis with arthritis may occur in many systemic autoimmune diseases, including spondyloarthritis, juvenile idiopathic arthritis, Behçet's disease and sarcoidosis. Recent advances in the field of autoimmune diseases have provided new insights and developments of diverse therapies for noninfectious uveitis. However, the optimal treatment strategy for patients with uveitis remains to be defined. Topical and/or systemic corticosteroids are the first-line therapy of autoimmune uveitis, but relapses are frequent after treatment cessation. Chronic use of corticosteroids is associated with significant systemic and ocular side effects. Many patients require treatment with conventional immunomodulatory drugs and/or biological agents in order to maintain long-term remission for both ocular inflammation and systemic disease. Treatment of uveitis should be individualized based on several factors, including the location of ocular inflammation, the inflammation severity, the impact on visual function and quality of life, the type of systemic autoimmune disease, and comorbidities.

This paper provides a concise overview of the current treatment modalities and strategies available for adult patients with uveitis associated with systemic autoimmune diseases.
\end{abstract}

Keywords: autoimmune uveitis, corticosteroids, immunomodulatory agents, biological therapy

\section{BACKGROUND}

Uveitis is traditionally defined as an inflammatory process of the uveal tract (iris, cilliary body and choroids). However, the inflammatory process can affect its adjacent structures as well, including vitreous humour, retina, optic nerve and vessels. Thereafter, the term "uveitis" can be used to describe intraocular inflammation in general $(1,2)$. The International Uveitis Study Group (IUSG) established a diagnostic system based primarily on the location of the intraocular inflammatory process. Inflammation predominantly of the iris or ciliary body is termed anterior uveitis (iritis, iridocyclitis and anterior cyclitis). The term intermediate uveitis is used for inflammation primarily in the vitreous body (vitritis), but also in pars plana (pars planitis) and ciliary body (posterior cyclitis). Posterior uveitis affects the choroid and possibly the retina (choroiditis, retinochoroiditis and chorioretinitis). Panuveitis encompasses inflammation of the anterior chamber, vitreous and retina or choroid (3). Acute anterior uveitis is the most common form of uveitis, followed by posterior, panuveitis and intermediate uveitis (4). For a complete definition of the pattern of uveitis, a classification system was defined by SUN (Standardization of Uveitis Nomenclature), that take into consideration besides anatomical location, the following parameters: onset (acute or insidious), duration (limited or persistent), clinical course (acute, chronic or recurrent) and laterality (unilateral or bilateral). In addition, according to the grading system defined by the SUN group, it is possible to quantify the amount of cells and flare seen at slit lamp and indirect ophtalmoscope examination in the anterior chamber. Grading the level of inflammation is helpful in initiating and monitoring the response to treatment (5).

In terms of etiology, uveitis can be either infectious or noninfectious. Non-infectious uveitis, can be due to immune-mediated systemic diseases, ocular immune-mediated syndromes, trauma, medications, or can be idiopathic. More than $25 \%$ of patients with uveitis have systemic autoimmune diseases, such as spondyloarthritis ( $\mathrm{SpA})$, juvenile 
idiopathic arthritis (JIA), Behçet's disease, sarcoidosis, systemic lupus erythematosus, granulomatosis with poliangiitis, chronc inflammatory bowel diseases, Vogt-Koyanagi-Harada disease, multiple sclerosis $(6,7)$. In a retrospective study comprising 4911 patients from a US tertiary uveitis service, the systemic diseases most commonly associated with uveitis were sarcoidosis (7\%), SpA (5\%), JIA (5\%) and Behçet's disease (3\%) (8). Non-infectious chronic uveitis is associated with high risk of ocular complications, including cystoid macular edema, cataract, increased intraocular pressure/glaucoma and retinopathy. Approximately $10 \%$ of legal blindness is caused by uveitis and its complications (9). Moreover, uveitis has a negative impact on the quality of life (10).

The clinical examination is the cornerstone of the diagnosis of uveitis. Specific patterns of uveitis are frequently associated with systemic diseases. The coexistence of unilateral acute anterior uveitis with inflammatory arthritis strongly suggests SpA. In contrast, anterior uveitis in patients with Behçet's disease, JIA and sarcoidosis are usually chronic and affect both eyes. Moreover, patients with Behçet's disease, JIA and sarcoidosis frequently have intermediate, posterior or panuveitis, with a worse visual prognosis. Other semiological features provide clues for etiologic diagnosis. For example, in sarcoidosis can occur granulomatous lesions (large keratic precipitates at ophtalmoscopy or nodules in the iris) and retinal venous vasculitis. In contrast, uveitis in $\mathrm{Be}-$ hçet's disease is never granulomatous, and in rare cases may be associated with retinal arterial vasculitis (7).

\section{PRINCIPLES OF UVEITIS THERAPY}

Recent advances in the field of autoimmune diseases and ocular inflammatory disease have provided new insights and developments of diverse therapies. Currently, there are many treatment options for autoimmune uveitis, including corticosteroids, various conventional immunomodulatory drugs and biologic agents. However, the evidence of their efficacy and safety in uveitis is limited, as the majority of studies in the field are small case series, case-control or cohort studies, with very few randomized control studies available. In clinical practice, the majority of immunomodulatory agents are used off-label in uveitis and treatment strategies are based on expert opinion and consensus guidelines $(4,11)$.
Treatment goals in autoimmune uveitis include suppression of inflammation, prevention of further structural damage, minimization of treatment side effects and preservation or restoration of visual function. In clinical practice, a "step-ladder approach" is often used, in order to maximize the clinical response while minimizing adverse effects. According to current guidelines, corticosteroids are the first-line therapy for patients with active autoimmune uveitis. Corticosteroids are very efficient in controlling acute inflammation. However, chronic corticosteroid treatment carries significant systemic and ocular side effects. Immunomodulatory drugs have shown good efficacy in controlling ocular inflammation, preventing uveitis relapses, and restoring or preserving of visual function. Their use as steroid-sparing agents has shown promising results in maintain long-term remission for both ocular inflammation and systemic disease. Moreover, immunomodulatory therapy has a more favorable long-term safety profile compared with chronic corticosteroid use $(4,12)$.

Treatment strategy depends on the type of uveitis (based on anatomical location and laterality) and severity of inflammation. Acute anterior uveitis usually has a good prognosis compared with other forms of uveitis and respond well to symptomatic treatment with topical corticosteroids (eye drops) and mydriatic agents (for prevention of posterior sinechia). In severe inflammation or in non-compliant patients are indicated periocular injections or systemic corticosteroids. Steroid-sparing immunomodulatory therapy, such as sulfasalazine, methotrexate or anti-TNFa agents could be efficient therapeutic alternatives for patients who failed to respond to corticotherapy. In cases of intermediate and posterior uveitis, topical corticosteroids have poor penetration of the vitreous, thereafter are not sufficient to control eye inflammation. The laterality and the etiology of uveitis are factors that influence the choice of treatment modalities. Acute attack of unilateral uveitis may be treated with periocular injections of corticosteroids, while systemic corticotherapy is preferred when bilateral lesions are present. The maintenance therapy may be done with a biodegradable corticosteroid intravitreal implant, but in patients with more severe inflammation or in the presence of a systemic autoimmune disease a low dose of systemic corticosteroid may be necessary. Patients with recurrent or refractory uveitis require addition of immunomodulatory agents. However, immunomodulatory 
treatment administered for the underlying systemic autoimmune disease may ameliorate the eye inflammation $(1,4,7,13)$.

\section{THERAPEUTIC OPTIONS FOR AUTOIMMUNE UVEITIS}

Several effective therapeutic options are available for treatment of autoimmune uveitis, although standard therapeutic regimen are still lacking. Corticosteroids and immunomodulatory agents play a central role in achieving sustained control of ocular inflammation, and thereafter in preservation of visual function.

\section{CORTICOSTEROIDS}

Corticosteroids have been the mainstay of therapy in autoimmune uveitis since 1950. Corticosteroids are valuable symptomatic drugs, very efficient in controlling acute attacks of uveitis or flares of chronic uveitis, but relapses are frequent after treatment cessation. However, chronic corticotherapy is not appropriate for maintaining remission, because it is not curative and is associated with many ocular and systemic adverse events, including secondary glaucoma, cataract, osteoporosis, Cushing syndrome and diabetes (4). In order to be effective, corticosteroids should be used aggressively during the initial phase of therapy and should be tapered gradually over a period of months to prevent relapses (7).

Topical therapy with high-potency steroids (e.g. prednisolone acetate $1 \%$ ) is the first-line treatment recommended in common acute anterior uveitis (e.g. associated with SpA). Dosing frequency should be tailored according to the severity of the anterior segment inflammation and treatment duration should be at least 6-8 weeks. To evaluate the response to treatment, patients should be monitored weekly for quantification of ocular inflammation (e.g. positive response when there is a two-grade improvement in ocular inflammation). Treatment should be stopped only after complete resolution of all cells, flare and macular edema $(1,7)$.

Local corticosteroid injections (subconjunctival or intravitreal) with triamcinolone acetonid are recommended in unilateral intermediate uveitis associated with decreased vision or with macular edema, in severe acute anterior uveitis, as an adjuvant in posterior uveitis and in patients non-compliant with topical treatment (2). Many patients with chronic intermediate or posterior uveitis with frequent relapses or macular edema may benefit from an intravitreal implant (with dexamethasone or fluocinolon acetat). Intraocular pressure should be closely monitored, as the use of local corticosteroids further increases the risk of glaucoma $(14,15)$.

Systemic corticotherapy is recommended in vision threatening uveitis, such as bilateral severe posterior uveitis or panuveitis (e.g. associated with Behçet's disease and sarcoidosis), or chronic refractory acute anterior uveitis There is no standard regimen for this treatment, the attack dose, duration of treatment and taper plans may vary according to clinical response and tolerability. A commonly recommended attack dose of oral prednisone is $1 \mathrm{mg} / \mathrm{kg}$ daily for up to 1 month, followed by slow taper $(5-10 \mathrm{mg} /$ week) $(1,4,7)$. "Rescue therapy" with high dose intravenous pulses of methylprednisolone is recommended in severe attacks of sight-threatening inflammation, like retinal vasculitis in Behçet's disease. Many patients with autoimmune uveitis need long-term maintenance corticotherapy. According to expert opinion, the maintenance dose should not exceed $10 \mathrm{mg}$ oral prednisone equivalent per day. Adjunctive immunomodulatory therapy is strongly recommended in patients with chronic and severe uveitis requiring steroid doses greater than 7.5-10 mg/day for controlling ocular inflammation (16).

\section{IMMUNOMODULATORY DRUGS}

Immunomodulatory drugs are widely used as corticosteroid-sparing agents in rheumatology and other autoimmune diseases. In recent years, this pharmacologic strategy has been translated to the treatment of ocular inflammation. Studies indicate that treatment with both conventional immunomodulatory drugs and biological agents results in better outcome for uveitic patients (17-21). Current guidelines recommend immunomodulatory drugs in the following settings: as corticosteroid-sparing agents in order to achieve sustained control of inflammation after tapering corticosteroids, in uveitis with insufficient response to primary corticosteroid therapy, in cases with frequent relapses (more than three flares in a year or more than two flares in less than 3 months), and in patients with contraindications or intolerance to systemic corticotherapy. In addition, early administration (first-line treatment) of immunomodulatory drugs is recommended for management of specific diseases characterized by severe 
ocular inflammation and poor response with lower levels of corticotherapy: Behçet's disease with retinal vasculitis, ANCA positive systemic vasculitis (e.g. granulomatosis with poliangiitis), Vogt-Koyanagi Harada's disease, and severe intermediate, posterior uveitis or panuveitis in JIA and sarcoidosis. Patients should be closely monitored for clinical response, which should be observed within 3 months of treatment. Treatment withdrawal should not be attempted before the ocular inflammation has been in stable and complete remission for at least 2 years $(12,16,22,23)$.

\section{CONVENTIONAL IMMUNOMODULATORY DRUGS}

There are three main classes of conventional immunomodulatory drugs that are used in uveitis treatment: calcineurin inhibitors (cyclosporine, tacrolimus), antimetabolites (methotrexate, azathioprine and mycophenolate mofetil), and alkylating agents (cyclophosphamide and chlorambucil). In the retrospective multicenter SITE study (Systemic Immunosuppressive Therapy for Eye diseases), conventional immunomodulatory drugs were modestly effective for controlling inflammation and for achieving corticosteroid-sparing objectives. Regarding the safety profile, the same study concluded that these agents represent safe therapeutic alternatives in the treatment of chronic or recurrent uveitis (24). Cyclosporine, methotrexate, azathioprine and mycophenolate mofetil are the most frequently used conventional immunomodulatory drugs for the treatment of severe or refractory autoimmune uveitis. The choice of immunomodulatory agent depends on the underlying systemic disease and the characteristics of the patient (age, sex, comorbidities, compliance) (1).

Cyclosporine in doses of 150-250 mg/day has been shown to effectively control ocular inflammation in refractory uveitis of various etiologies, including Behçet's disease, sarcoidosis, Vogt-Koyanagi Harada's disease, as well as in idiopathic retinal vasculitis. In the SITE study, $52 \%$ of the 373 patients treated with cyclosporine gained sustained remission of ocular inflammation, and tapering corticosteroids succeeded in $36 \%$ of patients by 1 year (24). In controlled studies of patients with recurrent uveitis in Behçet's disease, cyclosporine was proven to be superior over colchicine or chlorambucil (25). Tacrolimus showed comparable efficacy with cyclosporine, with a better safety profile (4).
Methotrexate has been the longest studied drug in the treatment of uveitis of different etiologies. It is indicated in the majority of types of ocular inflammation, including anterior, intermediate, posterior uveitis and panuveitis. Similar to its administration in rheumatic diseases, methotrexate is commonly administered intermittently at low-doses $(7.5-25 \mathrm{mg} /$ $\mathrm{kg}$ per week), and it can be titrated according to clinical response at a maximum dose of $50 \mathrm{mg} /$ week (26). In several retrospective studies, methotrexate it was shown to be moderately efficient for management of ocular inflammation, the best results being obtained for anterior uveitis and scleritis (4). In the SITE study, $66 \%$ out of 384 patients treated with methotrexate were in remission at 1 year, allowing for tapering of corticosteroids in $58 \%$ of patients (24). In a randomized trial, methotrexate showed a higher response rate than mycophenolate mofetil, but no differences were found in terms of corticosteroid sparing success (26).

Mycophenolate mofetil has been used in monotherapy or associated with other immunomodulatory agents in the treatment of ocular inflammation. It is administered orally in doses up to $3 \mathrm{~g}$ a day (27). In the SITE study there were 236 patients treated with mycophenolate mofetil, of whom $73 \%$ obtained remission at 1 year, while $55 \%$ of patients were able to cease corticotherapy (24).

Azathioprine at a standard dose of $2-3 \mathrm{mg} / \mathrm{kg} /$ day, has been proven to be effective in management of a wide range of ocular inflammatory diseases, including JIA and Behçet's disease. In a retrospective cohort, it was suggested that azathioprine might be especially effective for patience with intermediate uveitis (17). In the SITE study, which included 145 patients treated with azathiopirine, control of ocular inflammation was obtained in $62 \%$ of patients and a corticosteroid-sparing effect in $47 \%$ of patients, at 1 year (24).

Cycophosphamide and Chlorambucil use is generally restricted to severe sight-threatening uveitis, mainly those associated with Behçet's disease $(1,27)$.

\section{BIOLOGIC AGENTS}

Biologic agents are widely used in rheumatology, and recently have been used with good clinical results for treatment of autoimmune uveitis resistant to conventional immunomodulatory drugs. In the context of uveitis associated with systemic autoimmune disorder, biologic therapy might induce long-term 
remission for both ocular inflammation and systemic disease (11). Currently, a wide spectrum of biologics is available, including anti-cytokine monoclonal antibodies (e.g. anti-TNFa, anti-IL6, anti-IL1, anti-IL17, anti-IL12/23), anti-soluble receptor antibodies (e.g. anti-receptor of TNFa), interferons, B-cell inhibitors (e.g. rituximab), anti-vascular endothelial growth factor antibodies (e.g. bevacizumab) (2). Since the first report on efficacy of anti-TNFa in refractory posterior uveitis (28), these agents have been increasingly used in clinical practice. At present, there are substantial data regarding the efficacy and safety of anti-TNFa in autoimmune uveitis and recommendations are available for their use (11).

In controlled studies, anti-tumor necrosis factor (TNF)-a antibodies were proven to be highly effective in uveitis associated with systemic autoimmune diseases (29-32) In a recent review comprising more than 1000 patients with refractory uveitis it was shown that infliximab and adalimumab are effective in the treatment of autoimmune uveitis, while etanercept seems to be ineffective (33). Moreover, the occurrence of uveitis in patients that never had eye involvement or the relapse of uveitis was described in patients treated with anti-TNFa agents. Several reports have shown that the frequency of these paradoxical uveitis is higher in patients treated with etanercept when compared to that observed in patients treated with monoclonal antibodies (infliximab and adalimumab) (11). In a multicentre study from French Uveitis Network, anti-TNFa agents were highly effective in the treatment of refractory uveitis (main etiologies included Behçet's disease, JIA, SpA and sarcoidosis), with improvement in $87 \%$ and $93 \%$ of patients at 6 months and 12 months, respectively (34). Moreover, in univariate analysis was shown that an underlying diagnosis of Behçet's disease and number of previous uveitis flares were factors associated with complete anti-TNFa treatment response. A systematic review of the efficacy of anti-TNFa in uveitis associated with Behçet's disease showed a rapid and sustained control of uveitis in $89 \%$ and $100 \%$ of patients treated with infliximab and adalimumab, respectively (35).

According to the expert panel, anti-TNFa agents are strongly recommended as second-line agents in the treatment of refractory and recurrent uveitis associated with JIA and SpA. In vision-threatening uveitis associated with Behçet's disease, monoclonal antibodies may be recommended as first-line agents, in order to achieve a quick clinical response. Other possible indications of monoclonal antibodies (infliximab and adalimumab) as second-line agents include severe forms of ocular inflammation in sarcoidosis, scleritis and panuveitis. According to available data, the experts strongly recommend that use of monoclonal antibodies (infliximab and adalimumab) should be considered before etanercept for ocular inflammation (11). More recently, the efficacy and safety of adalimumab in the treatment of sight-threatening refractory uveitis have been confirmed in two randomized prospective controlled clinical trials (VISUAL 1 and VISUAL 2). The results of these studies have resulted in adalimumab being the first non-corticosterod licensed treatment of noninfectious intermediate and posterior uveitis or panuveitis $(36,37)$.

Many new biologics (e.g. rituximab, abatacept, tocilizumab, alemtuzumab) have been used as "rescue therapies" in small case-series of patients with autoimmune uveitis refractory to previous conventional immunomodulators and/or anti-TNFa agents. According to a recent systematic review, a successful treatment outcome can be achieved in many patients (38). However, due to lack of adequate clinical trials, the utility of these new biologics in clinical practice cannot be currently established (3).

\section{CONCLUSIONS}

Uveitis comprises a heterogenous group of sight-threatening immune-mediated ocular disorders, which in many cases are associated with systemic autoimmune diseases. The pattern of uveitis (according to location, clinical course and laterality) and underlying systemic disease are the main factors that must be taking into account when deciding treatment strategies. Corticosteroids are the first-line therapy for patients with active uveitis. Many patients require treatment with conventional immunomodulatory drugs and/or biological agents in order to maintain long-term remission for both ocular inflammation and systemic disease. Further assessment of currently used immunomodulatory agents, as well as emerging drugs is needed to provide more evidence for the efficacy and safety of these drugs and to establish the optimal treatment strategy of autoimmune uveitis. 


\section{REFERENCES}

1. Chora I., Borges T., Dias C. et al. Clinical management of uveitis in systemic rheumatic autoimmune diseases in adults. Autoimmun. Infec. Dis. 2015; doi http://dx.doi.org/10.16966/2470-1025.104.

2. Mérida S., Palacios E., Navea A. et al. New immunosuppressive therapies in uveitis treatment. Int. J. Mol. Sci. 2015; 16:1877818795.

3. Bloch-Michel E., Nussenblatt R.B. International uveitis study group recommendations for the evaluation of intraocular inflammatory disease. Am. J. Ophthalmol. 1987;103:234-235.

4. Barry J.R., Nguyen Q.D., Lee R.W. et al. Pharmacotherapy for uveitis: current management and emerging therapy. Clinical Ophtalmol. 2014; 8:1891-1911.

5. Jabs D.A., Nussenblatt R.B., Rosenbaum J.T. Standardization of uveitis nomenclature for reporting clinical data. Results of the First International Workshop. Am. J. Ophthalmol. 2005;140:509-516.

6. Wakefield D, Chang JH Epidemiology of uveitis. Int. Ophthalmol. Clin. 2005; 45:1-13

7. Sève P., Kodjikian L., Adélaïde L. et al. What do rheumatologists need to know?. Joint Bone Spine 2015. doi: http://dx.doi. org/10.1016/j.jbspin.2015.06.002.

8. Zaidi A.A., Ying G., Daniel E. et al. Hypopyon in patients with uveitis. Ophthalmology. 2010; 117:366-372.

9. Rothova A., Suttorp-van Schulten M.S., Frits Treffers W. et al. Causes and frequency of blindness in patients with intraocular inflammatory disease. Br. J. Ophthalmol. 1996; 80:332-336.

10. Murphy C.C., Hugh E.H., Frost N.A. et al. Quality of life and visual function in patients with intermediate uveitis. Br. J. Ophtalmol. 2005, 89:1161-1165.

11. Levy-Clarke G., Jabs D.A., Read R.W. et al. Expert panel recommendations for the use of anti-tumor necrosis factor biologic agents in patients with ocular inflammatory disorders. Ophtalmology. 2014; 121:785-796.

12. Lee K., Bajwa A., Freitas-Neto C.A. et al. A comprehensive review and update on the non-biologic treatment of adult noninfectious uveitis: part I. Expert Opin. Pharmacother. 2014; 15:2141-2154.

13. Fabiani C., Vitale A., Lopalco G. et al. Different roles of TNF inhibitors in acute anterior uveitis associated with ankylosing spondylitis: state of the art. Clin. Rheumatol. 2016; 35:2631-2638.

14. Kempen J.H., Altaweel M.M., Holbrook J.T. et al. The multicenter uveitis steroid treatment trial: rationale, design, and baseline characteristics. Am. J. Ophthalmol. 2010; 149:550-561.

15. Lowder C., Belfort R. Jr, Lightman S. et al. Ozurdex HURON Study Group. Dexamethasone intravitreal implant for noninfectious intermediate or posterior uveitis. Arch. Ophthalmol. 2011; 129:545553

16. Jabs D.A., Rosenbaum J.T., Foster S. et al. Guidelines for the use of immunosuppressive drugs in patients with ocular inflammatory Disorders: recommendations of an expert panel. Am. J. Ophthalmol. 2000; 130:492-513.

17. Pasadhika S., Kempen J.H., Newcomb C.W. et al. Azathioprine for ocular inflammatory diseases. Am. J. Ophthalmol. 2009; 148:500-509.

18. Gangaputra S., Newcomb C.W., Liesegang T.L. et al. Methotrexate for ocular inflammatory diseases. Ophthalmology 2009; 116:2188-2198.

19. Kaçmaz R.O., Kempen J.H., Newcomb C. et al. Cyclosporine for ocular inflammatory diseases. Ophthalmology 2010; 117:576-584.

20. Durrani K., Zakka F.R., Ahmed M. et al. Systemic therapy with conventional and novel immunomodulatory agents for ocular inflammatory disease. Surv. Ophthalmol. 2011; 56:474-510.
21. Heo J., Sepah Y.J., Yohannan J. et al. The role of biologic agents in the management of non-infectious uveitis. Expert Opin. Biol. Ther. 2012; 12:995-1008.

22. Muñoz-Fernández S., Martín-Mola E. Uveitis. Best Pract. Res. Clin. Rheumatol. 2006; 20:487-505.

23. Foster C.S., Kothari S., Anesi S.D. et al. The ocular immunology and uveitis foundation preferred practice patterns of uveitis management. Surv. Ophthalmol. 2016; 61:1-17.

24. Kempen J.H., Daniel E., Gangaputra S. et al. Methods for identifying long-term adverse effects of treatment in patients with eye diseases: the Systemic Immunosuppressive Therapy for Eye Diseases Cohort Study. Ophthalmic Epidemiol. 2008; 15:47-55.

25. Pato E., Muñoz-Fernández S., Francisco F. et al. Systematic review on the effectiveness of immunosuppressants and biological therapies in the treatment of autoimmune posterior uveitis. Semin. Arthritis. Rheum. 2011; 40:314-323.

26. Rathinam S.R., Babu M., Thundikandy R. et al. A randomized clinical trial comparing methotrexate and mycophenolate mofetil for noninfectious uveitis. Ophtalmology 2014; 121:1863-1870.

27. Castiblanco C., Foster C.S. Review of systemic immunosuppression for autoimmune Uveitis. Ophthalmol.Ther. 2014; 3: 17-36

28. Murphy C.C.,Greiner K., Plskova J. et al. Neutralizing tumor necrosis factor activity leads to remission in patients with refractory noninfectious posterior uveitis. Arch. Ophthalmol. 2004; 122:845-851

29. Diaz-Llopis M., Salom D., Garcia-de-Vicuña C. et al. Treatmentof refractory uveitis with adalimumab: a prospective multicenter study of 131 patients. Ophthalmology. 2012; 119:1575-1581.

30. Okada A.A., Goto H., Ohno S. et al. Multicenter study of infliximab for refractory uveoretinitis in Behcet disease. Arch.Ophthalmol. 2012; 130:592-598.

31. Dobner B.C., Max R., Becker M.D. et al. A three-centre experiencewith adalimumab for the treatment of noninfectiousuveitis. Br. J. Ophthalmol. 2013; 97:134-138.

32. Kruh J.N., Yang P., Suelves A.M. et al. Infliximab for the treatment of refractory noninfectious uveitis: a study of 88 patients with longterm follow-up. Ophthalmology. 2014; 121:358-364

33. Cordero-Coma M., Yilmaz T., Onal S. Systematic review of antitumor necrosis factor- $\alpha$ therapy for treatment of immune-mediated uveitis. Ocul. Immunol. Inflamm. 2013; 21:19-27.

34. Vallet H., Seve P., Biard L. Infliximab versus Adalimumab in the treatment of refractory inflammatory uveitis: A multicenter study from the french uveitis network. Arthritis Rheumatol. 2016; 68:1522-1530

35. Arida A., Fragiadaki K., Giavri E. et al. Anti-TNF agents for Behçet's disease: analysis of published data on 369 patients. Semin. Arthritis Rheum. 2011; 41:61-70.

36. Jaffe G.J.J., Dick A.D., Brézin A.P. et al. Adalimumab in Patients with Active Noninfectious Uveitis. N. Engl. J. Med. 2016; 375:932-943.

37. Nguyen Q.D., Merill P.T., Jaffe G.J. et al. Adalimumab for prevention of uveitic flare in patients with inactive non-infectious uveitis controlled by corticosteroids (VISUAL II): a multicentre, double-masked, randomised, placebo-controlled phase 3 trial. Lancet. 2016; 388:1183-1192.

38. Simonini G., Cimaz R., Jones G.T., Macfarlane G.J. Non-antiTNF biologic modifier drugs in non-infectious refractory chronic uveitis: The current evidence from a systematic review. Semin. Arthritis. Rheum. 2015; 45:238-250. 